\title{
Time-dependent postmortem redistribution of butyrfentanyl and its metabolites in blood and alternative matrices in a case of butyrfentanyl intoxication
}

\author{
Staeheli, Sandra N ; Baumgartner, Markus R ; Gauthier, Saskia ; Gascho, Dominic ; Jarmer, Juliane ;
} Kraemer, Thomas ; Steuer, Andrea E

\begin{abstract}
A fatal case of butyrfentanyl poisoning was investigated at the Zurich Institute of Forensic Medicine. At admission at the institute approx. $9 \mathrm{~h}$ after death (first time point, t1), femoral and heart blood (right ventricle) was collected, as well as samples from the lung, liver, kidney, spleen, muscle and adipose tissue using computed tomography (CT)-guided biopsy sampling. At autopsy (t2), samples from the same body regions were collected manually. Additionally, urine, heart blood (left ventricle), gastric content, brain samples and hair were collected. Butyrfentanyl concentrations and relative concentrations of the metabolites carboxy-, hydroxy-, nor-, and desbutyrfentanyl were determined by LC MS/MS and LC-QTOF. At t1, butyrfentanyl concentrations were $66 \mathrm{ng} / \mathrm{mL}$ in femoral blood, $39 \mathrm{ng} / \mathrm{mL}$ in heart blood, $110 \mathrm{ng} / \mathrm{g}$ in muscle, $57 \mathrm{ng} / \mathrm{g}$ in liver, $160 \mathrm{ng} / \mathrm{g}$ in kidney, $3100 \mathrm{ng} / \mathrm{g}$ in lung, $590 \mathrm{ng} / \mathrm{g}$ in spleen and $550 \mathrm{ng} / \mathrm{g}$ in adipose tissue. At t2, butyrfentanyl concentration in urine was $1100 \mathrm{ng} / \mathrm{mL}$, in gastric content $2000 \mathrm{ng} / \mathrm{mL}$, in hair 11,000 pg/mg and brain concentrations ranged between $200340 \mathrm{ng} / \mathrm{g}$. Carboxy- and hydroxybutyrfentanyl were identified as most abundant metabolites. Comparison of t1 and t2 showed a concentration increase of butyrfentanyl in femoral blood of $120 \%$, in heart blood of $55 \%$ and a decrease in lung of $30 \%$ within $19 \mathrm{~h}$. No clear concentration changes could be observed in the other matrices. Postmortem concentration changes were also observed for the metabolites. In conclusion, butyrfentanyl seems to be prone to postmortem redistribution processes and concentrations in forensic death cases should be interpreted with caution.
\end{abstract}

DOI: https://doi.org/10.1016/j.forsciint.2016.05.034

Posted at the Zurich Open Repository and Archive, University of Zurich

ZORA URL: https://doi.org/10.5167/uzh-124774

Journal Article

Accepted Version

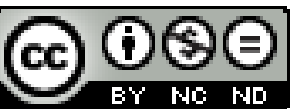

The following work is licensed under a Creative Commons: Attribution-NonCommercial-NoDerivatives 4.0 International (CC BY-NC-ND 4.0) License.

Originally published at:

Staeheli, Sandra N; Baumgartner, Markus R; Gauthier, Saskia; Gascho, Dominic; Jarmer, Juliane; Kraemer, Thomas; Steuer, Andrea E (2016). Time-dependent postmortem redistribution of butyrfentanyl 
and its metabolites in blood and alternative matrices in a case of butyrfentanyl intoxication. Forensic Science International, 266:170-177.

DOI: https://doi.org/10.1016/j.forsciint.2016.05.034 


\section{Time-dependent postmortem redistribution of butyrfentanyl and its metabolites in blood and alternative matrices in a case of butyrfentanyl intoxication}

Sandra N. Staeheli ${ }^{a}$, Markus R. Baumgartner ${ }^{b}$, Saskia Gauthier ${ }^{c}$, Dominic Gascho $^{c}$, Juliane Jarmer ${ }^{c}$, Thomas Kraemer $^{a}$, Andrea E. Steuer ${ }^{a}$

aDepartment of Forensic Pharmacology \& Toxicology, Zurich Institute of Forensic Medicine, University of Zurich, Switzerland

${ }^{\mathrm{b}}$ Center for Forensic Hairanalytics, Zurich Institute of Forensic Medicine, University of Zurich, Switzerland

'Department of Forensic Medicine \& Imaging, Zurich Institute of Forensic Medicine, University of Zurich, Switzerland

Corresponding author:

Dr. Andrea E. Steuer University of Zurich

Zurich Institute of Forensic Medicine (ZIFM)

Department of Forensic Pharmacology \& Toxicology

Winterthurerstrasse 190/52

CH-8057 Zurich

Switzerland

Tel.: 0041 446355679; fax: 0041446356852

E-mail address: andrea.steuer@irm.uzh.ch

Formal publication is available in Forensic Science International, vol. 266, pp 170-177; at https://doi.org/10.1016/j.forsciint.2016.05.034

(C) 2020. This manuscript version is made available under the CC-BY-NC-ND 4.0 license http://creativecommons.org/licenses/by-nc-nd/4.0/ 


\begin{abstract}
A fatal case of butyrfentanyl poisoning was investigated at the Zurich Institute of Forensic Medicine. At admission at the institute approx. 9 hours after death (first time point, t1), femoral and heart blood (right ventricle) was collected, as well as samples from the lung, liver, kidney, spleen, muscle and adipose tissue using computed tomography (CT) -guided biopsy sampling. At autopsy (t2), samples from the same body regions were collected manually. Additionally, urine, heart blood (left ventricle), gastric content, brain samples and hair were collected. Butyrfentanyl concentrations and relative concentrations of the metabolites carboxy-, hydroxy-, nor-, and desbutyrfentanyl were determined by LC-MS/MS and LC-QTOF. At t1, butyrfentanyl concentrations were $66 \mathrm{ng} / \mathrm{mL}$ in femoral blood, $39 \mathrm{ng} / \mathrm{mL}$ in heart blood, $110 \mathrm{ng} / \mathrm{g}$ in muscle, $57 \mathrm{ng} / \mathrm{g}$ in liver, $160 \mathrm{ng} / \mathrm{g}$ in kidney, $3100 \mathrm{ng} / \mathrm{g}$ in lung, $590 \mathrm{ng} / \mathrm{g}$ in spleen and $550 \mathrm{ng} / \mathrm{g}$ in adipose tissue. At t2, butyrfentanyl concentration in urine was $1100 \mathrm{ng} / \mathrm{mL}$, in gastric content $2000 \mathrm{ng} / \mathrm{mL}$, in hair $11000 \mathrm{pg} / \mathrm{mg}$ and brain concentrations ranged between 200-340 ng/g. Carboxy- and hydroxybutyrfentanyl were identified as most abundant metabolites. Comparison of $\mathrm{t} 1$ and $\mathrm{t} 2$ showed a concentration increase of butyrfentanyl in femoral blood of $120 \%$, in heart blood of 55\% and a decrease in lung of $30 \%$ within 19 hours. No clear concentration changes could be observed in the other matrices. Postmortem concentration changes were also observed for the metabolites. In conclusion, butyrfentanyl seems to be prone to postmortem redistribution processes and concentrations in forensic death cases should be interpreted with caution.
\end{abstract}

\title{
Keywords
}

Butyrfentanyl poisoning; time-dependent postmortem redistribution; LC-MS/MS; alternative matrices; biopsy, metabolite ratios 


\section{Introduction}

Butyrfentanyl belongs to the group of fentanyl-derived designer drugs which includes e.g. $\alpha$-methylfentanyl, 4fluorofentanyl, 3-methylfentanyl or acetylfentanyl. Increasing numbers of fentanyl consumption and occurrence of new fentanyl analogues have been observed [1-3], however mostly with little knowledge on pharmacological potency or pharmacokinetic and physicochemical properties. Also for butyrfentanyl, pharmacokinetic parameters are not known. They can only be assumed to be similar to fentanyl, which has a volume of distribution $\left(\mathrm{V}_{\mathrm{d}}\right)$ of 3-8 $\mathrm{L} / \mathrm{kg}$ and a pKa of 8.4 [4]. Butyrfentanyl potency ratio was found to be 7 compared to morphine and 0.13 compared to fentanyl [5]. Metabolism of fentanyl-derived designer drugs was investigated for 3-methylfentanyl and isofentanyl and was found to be similar to fentanyl, where the nor-metabolite shows highest abundance [6,7]. Prevalence or tissue distribution of butyrfentanyl metabolites has not been investigated to date, although a similar metabolism pathway to fentanyl and/or 3-methylfentanyl seems to be likely. Several poisoning cases with fentanyl analogues have been reported to date [2, 3, 8]; however, only few forensic case reports with butyrfentanyl involved are published and postmortem concentrations were presented only in one case [9-11]. Besides missing information on actual pharmacodynamic and -kinetic properties, interpretation of these postmortem concentrations needs to be done with great caution, as also information on postmortem redistribution (PMR) of butyrfentanyl does not exist so far. PMR complicates interpretation of forensic death cases with drugs involved. Several mechanisms such as e.g. drug degradation, new formation or diffusion processes can contribute to PMR and are believed to be time-dependent [12]. Basic drugs are believed to redistribute due to their increased ionization in the more acidic environment after death. Lipophilic drugs that have an apparent volume of distribution $\left(\mathrm{V}_{\mathrm{d}}\right)>3 \mathrm{~L} / \mathrm{kg}$, a high concentration ratio between central and peripheral blood ( $\mathrm{C} / \mathrm{P}$ ratio) or between the liver and peripheral blood (L/P ratio) are said to redistribute more than others due to their inhomogeneous distribution within the body at time of death [13-16]. However, prediction of PMR cannot be done only based on $\mathrm{V}_{\mathrm{d}}$ as the correlation is not very strong [17]. Data on time-dependent PMR is generally rare and mainly exists for classical drugs such as opiates, benzodiazepines, antidepressants or antipsychotics to date [18-20]. Time-dependent PMR of NPS has not been investigated so far. However, new psychoactive substances (NPS) are an emerging problem in forensic toxicology with many intoxication cases reported [1]. PMR of NPS can only be assumed based on structural similarities to known substances or estimated based on their physicochemical properties such as e.g. lipophilicity or pKa. Giving the structural similarities to fentanyl - a drug that is known for significant postmortem concentration increases in peripheral blood [21, 22] - PMR of butyrfentanyl seems to be likely. The aim of this work was to investigate tissue distribution and time-dependent PMR of butyrfentanyl and its main metabolites.

\section{Case history}

The dead body of a 23 year old male was found lying in the bathroom of his apartment. In his bedroom, a tray with traces of white powder and a tube was found. History of drug use was known. The external postmortem examination revealed no injuries or signs of a violent cause of death. The time of death was estimated as 2 to 4 hours prior to the medical examination. Further examination revealed few fresh appearing bruises on the right knee and the tibia, a single fresh abrasion on the base of the left neck and small fresh bleedings in the left temporal muscle and the left muscle between shoulder and tongue (omohyoid). Postmortem computed 
tomography (CT) imaging and the autopsy revealed few unspecific intoxication signs such as edema of the brain and a moderate full urinary bladder. Small amounts of residual white powder were found in the nose.

\section{Material and methods}

\section{Chemicals and reagents}

A methanolic solution $(1 \mathrm{mg} / \mathrm{mL})$ of $n$-butyrfentanyl was obtained from Cayman Chemical (delivered by Adipogen AG, Arlesheim, Switzerland) and a methanolic solution of the deuterated internal standard (IS, 0.1 $\mathrm{mg} / \mathrm{mL}$ ) fentanyl-d5 was obtained from Lipomed (Arlesheim, Switzerland). Water was purified with a Purelab Ultra millipore filtration unit (Labtech, Villmergen, Switzerland), acetonitrile of HPLC grade was obtained from Fluka (Buchs, Switzerland) and all other chemicals used were obtained from Merck (Zug, Switzerland).

\section{Postmortem samples}

Blood and alternative matrices were collected at two time points $\mathrm{t} 1$ and $\mathrm{t} 2$ after death according to [23]. After the routine CT imaging procedure (t1, 9 hours after death) on a 128-slice scanner (Somatom Definition Flash, Siemens Medical Solutions, Forchheim, Germany), introducer needles were placed into the right heart ventricle, the right lung, the right lobe of the liver, the right kidney, the spleen, subcutaneous adipose tissue of the waist, muscle tissue at the upper left thigh, the right femoral vein and the dural sac at the level of the lumbosacral junction using the virtobot system [23]. After placement of the introducer needles, another CT scan was performed to verify the needle positions. From the right heart ventricle and the femoral vein, $1 \mathrm{~mL}$ blood was collected using a $5 \mathrm{~mL}$ syringe. Cerebrospinal fluid was not available. Biopsies of approx. $22 \mathrm{~mm}$ length and 20 mg weight from tissues and organs were collected in triplicates from all matrices. Directly after sample collection, the biopsies were weighted into $2 \mathrm{~mL}$ Metal Bead Lysing Matrix tubes (MP Biomedicals, Illkirch, France). The body fluids were aliquoted in triplicates of $20 \mu \mathrm{L}$ into $2 \mathrm{~mL}$ Eppendorf Safe Lock Tubes (Schoenenbuch, Switzerland). The following day at autopsy (t2, 28 hours after death), samples from the same locations were collected where biopsies had been taken at t1. Additionally, heart blood from the left ventricle, urine, gastric content, a sample from the left lobe of the liver, cerebellum, frontal lobe, centrum semiovale, thalamus, praecuneus, a hair sample representing the full hair length $(2 \mathrm{~cm})$ from the occipital head region and a nose swab were collected. After autopsy, the solid matrices were aliquoted into triplicates of approx. $20 \mathrm{mg}$ and body fluids into triplicates of $20 \mu \mathrm{L}$. All samples were stored at $-20{ }^{\circ} \mathrm{C}$ until analysis.

\section{Routine systematic toxicological analysis}

Routine toxicological analysis was performed on femoral blood and urine collected at t2 (autopsy samples). Urine was screened initially by a cloned enzyme donor immunoassay (CEDIA®) for drugs of abuse (opiates, cocaine, cannabis, amphetamines, methadone, barbiturates, benzodiazepines, and lysergic acid diethylamide (LSD)) followed by a gas chromatography mass spectrometry (GC-MS) screening after enzymatic conjugate cleavage using Helix pomatia N., liquid-liquid extraction and semi-automatic data analysis in AMDIS adapted from Maurer/Pfleger/Weber [24]. Femoral blood was additionally screened for drugs by an untargeted LCMS/MS ion trap screening after simple protein precipitation (Bruker amazon®, Maurer/Wissenbach/Weber database [25]) and for ethanol and other volatile compounds by headspace GC flame ionization detector (HDGC-FID). Quantification of drugs in femoral blood was performed by LC-MS/MS [26]. 


\section{Sample preparation for PMR investigation}

Extraction of tissue and body fluid samples were performed according to Staeheli et al. [27]. Briefly, organ and tissue samples were homogenized using a Fast Prep®-24 Instrument (MP Biomedicals, Illkirch, France). To each sample, fentanyl-d5 (40 ng/mL) was added. Two liquid-liquid extractions (LLE) with butyl acetate/ethyl acetate $(1: 1, \mathrm{v} / \mathrm{v})$ were performed, one at $\mathrm{pH} 7.4$ and one at $\mathrm{pH} 13.5$. The extracts were combined, evaporated to dryness and reconstituted in $60 \mu \mathrm{L}$ mobile phase.

\section{LC-MS/MS analysis of butyrfentanyl}

The quantitative butyrfentanyl analysis of all samples was performed using a Thermo Fischer Ultimate 3000 UHPLC system (Thermo Fisher, San Jose, California, USA) coupled to a Sciex 5500 QTrap linear ion trap quadrupole MS (Sciex, Darmstadt/Germany). The MS was operated in the unscheduled multiple reaction monitoring (MRM) mode using eight transitions for butyrfentanyl. Four transitions corresponded to the ${ }^{12} \mathrm{C}$ isotope and four transitions corresponded to the ${ }^{13} \mathrm{C}$ isotope of butyrfentanyl. Quantifier transitions for butyrfentanyl and its ${ }^{13} \mathrm{C}$ isotope were $351 \rightarrow 188$ resp. $352 \rightarrow 189$, and $342 \rightarrow 188$ for fentanyl-d5. Remaining MS settings were according to [27]. An LC gradient elution was performed using a Phenomenex (Aschaffenburg, Germany) Synergy Polar RP column $(100 \times 2.0 \mathrm{~mm}, 2.5 \mu \mathrm{m})$ with $10 \mathrm{mM}$ ammonium formate buffer in water containing $0.1 \%(\mathrm{v} / \mathrm{v})$ formic acid $(\mathrm{pH} 3.5$, eluent $\mathrm{A})$ and acetonitrile containing $0.1 \%(\mathrm{v} / \mathrm{v})$ formic acid (eluent B) according to [27]. The MS was controlled by Analyst ${ }^{\circledR} 1.6 .2$ software and quantitation was performed with MultiQuant® 2.1.1 software. Butyrfentanyl concentrations in urine, lung, adipose tissue, gastric content and hair were calculated using the ${ }^{13} \mathrm{C}$ calibration, which avoided detector saturation problems. Concentrations of all other samples were calculated with the ${ }^{12} \mathrm{C}$ calibration. For the analysis of the hair sample, the calibration range was extended to $12000 \mathrm{pg} / \mathrm{mg}$ (not validated). Percentage concentration increase or decrease between $\mathrm{t} 1$ and $\mathrm{t} 2$ for each matrix was calculated. The concentration ratios between the matrices and peripheral blood were calculated for $\mathrm{t} 1$ and $\mathrm{t} 2$.

\section{LC-HRMS analysis of butyrfentanyl metabolites}

Investigation of the metabolites was performed on a Thermo Fischer Ultimate 3000 UHPLC system coupled to a Sciex 6600 QTOF System. The instrument was operated in the TOF MS mode with information dependent acquisition (IDA) of product ions. IDA criteria were set according to [28]. The LC start conditions were $10 \%$ eluent $\mathrm{B}$ for $1 \mathrm{~min}$ with a flow rate of $0.5 \mathrm{~mL} / \mathrm{min}, 2-18 \mathrm{~min}$ to $95 \% \mathrm{~B}$, hold for $1 \mathrm{~min}$ and $0.5 \mathrm{~min}$ reequilibrating to $10 \% \mathrm{~B}$. Injection volume was $10 \mu \mathrm{L}$. The MS was controlled by Analyst@ TF 1.7 software and data analysis was performed with PeakView ${ }^{\circledR} 2.1$ and MultiQuant ${ }^{\circledR} 2.1 .1$ software. LC-QTOF data was screened for the exact masses of butyrfentanyl, norbutyrfentanyl, carboxybutyrfentanyl, hydroxybutyrfentanyl and desbutyrfentanyl (Table 1). The metabolite relative concentrations were calculated by dividing the metabolite peak areas by the area of fentanyl-d5 (area ratio). Then the relative concentration changes between $\mathrm{t} 1$ and $\mathrm{t} 2$ were investigated. Additionally, the metabolite ratios were analyzed by dividing the relative concentrations (area ratios) of the metabolites by the mean relative concentration (area ratio) of butyrfentanyl in each matrix. 


\section{Hair analysis: sample preparation and LC-MS/MS analysis}

Extraction of the non-segmented hair sample was performed according to [29]. Before extraction, fentanyl-d5 (2500 pg/mg) was added as IS for the butyrfentanyl quantitation. Furthermore, the first wash solution (water) was also investigated after spiking the solution with the same IS and acidification. Then both samples, the extract of the lock of hair and the wash solution, were dried and reconstituted in $500 \mu \mathrm{L}$ mobile phase. Analysis on butyrfentanyl was performed by LC-MS/MS as described above. Hair analysis on other drugs was performed using validated routine LC-MS/MS methods which are not described in this case report.

\section{LC-MS/MS method validation}

The butyrfentanyl quantitation method was validated in terms of selectivity, matrix effects, accuracy, precision, calibration model and limit of quantitation in postmortem femoral blood according to [30].

\section{Selectivity}

Six blank postmortem blood samples from different sources and two blank postmortem blood samples spiked with IS (zero samples) were analyzed for interfering peaks with butyrfentanyl and fentanyl-d5.

\section{Calibration}

Ten calibrators were prepared in duplicates at concentrations 1, 5, 25, 100, 200, 500, 1000, 1500, 2000 and 2500 $\mathrm{ng} / \mathrm{mL}$. For calibrators 1-6 (1-500 ng/mL), the ${ }^{12} \mathrm{C}$ isotope of butyrfentanyl was used as quantifier. For calibrators 5-10 (200-2500 ng/mL) the ${ }^{13} \mathrm{C}$ isotope was used as quantifier. The regression lines for both calibrations ${ }^{12} \mathrm{C}$ and ${ }^{13} \mathrm{C}$ were calculated using a simple linear model with $1 / \mathrm{X}$ weighting to compensate for heteroscedasticity. Back calculation of the calibrator concentrations should result in less than $\pm 20 \%$ bias to the theoretical concentration.

\section{Accuracy and precision}

Six replicates of quality control (QC) samples at the concentrations levels QC low (2 ng/mL), QC med (300 $\mathrm{ng} / \mathrm{mL})$ and QC high $(2250 \mathrm{ng} / \mathrm{mL})$ were analyzed each on the same day. Concentration of QC low was calculated using ${ }^{12} \mathrm{C}$ calibration $(1-500 \mathrm{ng} / \mathrm{mL})$ and the concentration of $\mathrm{QC}$ high was calculated using ${ }^{13} \mathrm{C}$ calibration (200-2500 ng/mL). QC med concentration was determined with both calibrations. Accuracy was calculated as the percent deviation of the mean calculated concentration at each concentration level from the corresponding theoretical concentration. Precision was calculated as the relative standard deviation (RSD) within the QC levels.

\section{Matrix effect}

Matrix effect was investigated with six blank postmortem blood samples from different sources at the concentrations levels of QC low and QC high according to [30].

\section{Limits}

The limit of quantitation (LOQ) was defined as the concentration of the lowest calibrator ( $1 \mathrm{ng} / \mathrm{mL})$ fulfilling the criteria of signal to noise 10:1 and back-calculated concentrations within $+/-30 \%$ of theoretical concentration. The limit of detection (LOD) was not systematically investigated. 


\section{Results}

\section{Routine systematic toxicological analysis}

CEDIA® immunoassays in urine were positive for benzodiazepines and LSD. The LC-Ion Trap MS and the GC-MS screening revealed benzoylecgonine, hydroxymidazolam and a clarithromycin metabolite. Quantitative analysis in femoral blood gave negative results for cocaine, LSD and fentanyl. Benzoylecgonine and midazolam were below the limit of quantification of 1.25 and $11 \mathrm{ng} / \mathrm{mL}$, respectively and $1.0 \mathrm{ng} / \mathrm{mL}$ hydroxymidazolam was quantified. Ethanol or other volatiles were not detectable.

\section{LC-MS/MS method validation}

No interfering peaks were detected in the blank postmortem blood and zero samples. For both ${ }^{12} \mathrm{C}$ and ${ }^{13} \mathrm{C}$ calibration a linear regression model with $1 / \mathrm{X}$ weighting was used. $\mathrm{R}^{2}$ value was 0.99 for both calibrations. No difference between ${ }^{12} \mathrm{C}$ and ${ }^{13} \mathrm{C}$ calibration was observable for QC med. Validation results including accuracy, precision and matrix effects are given in Table 2.

\section{Butyrfentanyl analysis}

Butyrfentanyl concentrations were successfully determined in all samples and are displayed in Figure 1. At t1, highest concentrations of $3000 \mathrm{ng} / \mathrm{g}$ were found in lung tissue. Spleen and adipose tissue showed similar concentrations of 590 and $550 \mathrm{ng} / \mathrm{g}$, respectively. Lower concentrations were found in kidney $(160 \mathrm{ng} / \mathrm{g})$, muscle (110 ng/g), liver (57 ng/g), femoral blood (66 ng/mL) and heart blood (39 ng/mL). Concentration ratios between the matrices and femoral blood are given in Table 3. At t2, concentration in the left lobe of the liver was 30\% higher compared to the right lobe, although concentration ranges were overlapping. In gastric content $2000 \mathrm{ng} / \mathrm{mL}$ butyrfentanyl were measured. In brain tissue, mean concentrations of $200 \mathrm{ng} / \mathrm{g}$ were measured except for centrum semiovale, where the mean concentration was $340 \mathrm{ng} / \mathrm{g}$. A concentration change during the 19 hours between the two sampling time points was observed in peripheral blood $(+120 \%)$, heart blood $(+55 \%)$ and lung tissue $(-30 \%)$. Muscle, liver, kidney and adipose tissue showed mean concentration changes with overlapping concentration ranges between $\mathrm{t} 1$ and $\mathrm{t} 2$. Qualitative analysis (routine screening method) of the nose swab revealed butyrfentanyl.

\section{Butyrfentanyl metabolites}

The investigated butyrfentanyl metabolites were detected in all matrices at both time points except for norbutyrfentanyl, which in general showed low abundance and was not detected in adipose tissue at $\mathrm{t} 1$. At $\mathrm{t} 2$, norbutyrfentanyl was only detectable in urine, kidney and liver tissue. Next to the highest abundant peak, the extracted ion chromatogram of hydroxybutyrfentanyl indicated the presence of several potential minor isomers that were not further investigated. Present fragment ions of the highest abundant hydroxybutyrfentanyl peak in the MS/MS were 188.1436, 105.0694 and 281.2023.

The metabolite ratios (metabolite area/mean butyrfentanyl area) displayed in Figure 2 showed general highest metabolite abundance in urine, kidney and liver. In blood, carboxybutyrfentanyl was the most abundant metabolite at $\mathrm{t} 1$ followed by hydroxybutyrfentanyl. In tissues, hydroxybutyrfentanyl showed highest abundance followed by carboxybutyrfentanyl. Des- and norbutyrfentanyl were found in relatively low amounts in all matrices except for liver and kidney, where the metabolite ratios were higher. The metabolite ratios in different 
brain regions were rather homogeneous with carboxybutyrfentanyl as the lowest abundant metabolite after norbutyrfentanyl which was not detected. In Figure 2, metabolite ratios in the cerebellum are exemplarily displayed for all brain regions.

Comparison of the relative metabolite concentrations between $\mathrm{t} 1$ and $\mathrm{t} 2$ showed a decrease of carboxy- and norbutyrfentanyl in all matrices. Desbutyrfentanyl concentrations increased between $\mathrm{t} 1$ and $\mathrm{t} 2$ in blood and stayed on a similar level in other matrices. In contrast, hydroxybutyrfentanyl area ratios stayed on the same level in blood, but decreased in tissues. In Figure 3, the results are exemplarily displayed for blood, muscle, liver and kidney.

\section{Hair analysis}

In the hair sample $11000 \mathrm{pg} / \mathrm{mg}$ butyrfentanyl were detected and in the wash solution $3500 \mathrm{pg} / \mathrm{mg}$. Highest abundant metabolite was hydroxybutyrfentanyl with a metabolite ratio of 0.01 in the hair sample and 0.006 in the wash solution, respectively. Other metabolites showed a metabolite ratio in the hair extract below 0.002 . Further drugs detected were cocaine $(7600 \mathrm{pg} / \mathrm{mg})$, benzoylecgonine $(1200 \mathrm{pg} / \mathrm{mg})$, norcocaine $(160 \mathrm{pg} / \mathrm{mg})$, amphetamine (240 pg/mg), midazolam (20 pg/mg), $\alpha$-hydroxymidazolam (<LOQ), and ketamine (300 pg/mg).

\section{Discussion}

So far butyrfentanyl blood concentrations are reported only for few cases. In three non-lethal cases with mixed intoxications (combination with either fentanyl or acetylfentanyl) butyrfentanyl ranged from not detected to 0.9 $\mathrm{ng} / \mathrm{mL}$ in serum and from 2 to $66 \mathrm{ng} / \mathrm{mL}$ in urine [10]. In a fatal case, $58 \mathrm{ng} / \mathrm{mL}$ butyrfentanyl was measured in peripheral blood in combination with acetylfentanyl [9]. In our case, standard toxicological analysis did not reveal drugs in relevant concentrations. Standard toxicological analysis was unable to detect butyrfentanyl due to a missing library entry both in GC and LC-MS analysis. Only manual evaluation of the GC-MS urinary data revealed a mass of 145 representing a characteristic fragment ion of fentanyl, and careful mass spectra interpretation lead to an initial hint for butyrfentanyl. These findings could be further confirmed by manual evaluation of LC-Ion Trap data files and finally proven by comparison to a commercially available reference standard. The positive immunoassay result on LSD can probably be explained by cross reactivity with butyrfentanyl, as cross reactivity with fentanyl is already well known [31]. Missing competing causes of death (both toxicological and medicinal) lead to the conclusion, that an intoxication with butyrfentanyl was most probably the cause of death; thus, representing the first report on fatal butyrfentanyl monointoxication. The white powder residues in the nose turned out to be butyrfentanyl, which suggests sniffing of the drug as route of application. Hair analysis revealed a former drug history of cocaine, amphetamine, and ketamine over the past two months prior to death and proved the intake of low amounts of midazolam. Furthermore, the entire lock of hair was positive for butyrfentanyl at a level $11000 \mathrm{pg} / \mathrm{mg}$. However, a wash ratio (concentration of wash solution/concentration in hair) of 0.32 together with very low metabolic ratios for the different metabolites detected in hair and in the wash solution are indicators of a current sweat-mediated external contamination. This phenomenon is often observed in cases of acute opioid intoxication leading to death e.g. due to sweating during an agonal phase. 
A short one day method validation was performed for butyrfentanyl in postmortem blood as proposed by Peters and Drummer [30] for analysis of rarely occurring compounds. Validation exclusively in blood is of course generally not sufficient to evaluate the method performance on other matrices such as postmortem tissues. However, in the current study butyrfentanyl was included in an already existing method for 83 drugs in 11 different postmortem matrices. The former validation showed that accuracy and precision of the applied method was within the required ranges for the majority of the analytes in most matrices as long as an IS was used [27]. Only QC low precision exceeded the required limit minimally, which was considered as acceptable due to analysis of triplicate samples in the case investigation. In the presented study, calibration was done in postmortem blood instead of solvent, which should lead to a further improvement of accuracy. Comparison between time points was performed in a relative manner anyway. Therefore, validation only in postmortem blood was chosen as a compromise between extensive method validation and significance of the expected findings and the resulting accuracy and precision were considered as acceptable.

Distribution of butyrfentanyl within the body was inhomogeneous. The rather high concentration ratio between lung and femoral blood of 46 could be explained by the nasal application which may have led to inhalation of a part of the powder. Furthermore, the high perfusion rate of the lung in combination with lysosomal trapping may have been involved in the accumulation of butyrfentanyl in the lung as it is a rather lipophilic substance (calculated $\log \mathrm{P}$ of 4.44 by ALGOPS 2.1) with a $\mathrm{pK}_{\mathrm{a}}$ probably around 8 [32]. Gastric content showed high concentrations as well, which might have resulted from swallowing of powder after nasal application or secretion into the acidic gastric content after absorption. In brain, mean concentrations were approx. 1.5 times higher compared to femoral blood, except for the centrum semiovale, where the mean concentration was higher. As the centrum semiovale (white matter) has a higher lipid content compared to the other brain regions, butyrfentanyl may have showed an increased affinity to this brain region [33].

No time-dependent PMR data for fentanyl-derived designer drugs was available so far. However, studies on PMR of fentanyl revealed increases in postmortem concentrations in peripheral blood and no correlation between antemortem and postmortem blood concentrations [21, 34]. Butyrfentanyl concentration in femoral blood aligned with the concentrations in muscle tissue during the two sampling time points. Therefore, PMR in femoral blood may have been caused by butyrfentanyl diffusion from surrounding muscle tissue into femoral blood. The concentration increase in heart blood might have been caused by redistribution processes from the lung, where highest concentrations were detected. This redistribution mechanism may also explain the higher butyrfentanyl concentrations in the left heart ventricle compared to the right ventricle due to e.g. blood movement along the pulmonary veins. Also other adjacent tissues like heart muscle or stomach might have been involved; however, concentration in heart muscle was not determined. Butyrfentanyl heart to femoral blood concentration ratio of 0.6 at $\mathrm{t} 1$ in our case showed, that a $\mathrm{C} / \mathrm{P}$ ratio $<1$ might not always be a trustworthy criterion to exclude PMR processes within a case. Furthermore, liver to peripheral (L/P) ratio was $<1$ at both time points and does not support the thesis of drugs with L/P ratios < 5 being less prone to PMR [35], at least within a case. An explanation for the unexpected low concentrations in liver compared to blood might have resulted from a relatively fast death after intake of the substance and the resulting short time window for drug distribution and accumulation. However, the relatively high concentration in e.g. adipose tissue which has a rather low perfusion rate contradicts this theory. Whether these relatively high concentrations in adipose tissue 
resulted from the latest consumption or from an additional earlier intake cannot be differentiated. A general limitation of the study was lack of information on the postmortem interval between death and first sampling time point, which could not be analyzed due to practical reasons. Therefore no conclusions could be made on PMR in the first hours after death, which are believed to be a time interval in which relevant redistribution processes occur [36].

No information on butyrfentanyl metabolism and metabolite tissue distribution was available so far. Therefore, few phase I metabolites expected to occur in analogy to fentanyl [7] or 3-methylfentanyl [6] were included in the analysis of time-dependent PMR. Phase II metabolites were not investigated, as their extraction from the biological matrices could not be expected with the applied sample preparation. Due to lack of reference material, absolute quantification of the chosen metabolites was not possible and consequently, analytical recovery, accuracy and precision could not be determined. Estimations on abundance are based on area ratios relative to the parent drug, of course also not considering different ionization properties and MS response. Precision of extraction was partly covered by analysis of triplicates and revealed comparable results like butyrfentanyl itself and should therefore allow relative comparison between time points. The extracted ion chromatogram of $\mathrm{m} / \mathrm{z} 367.2380$ indicated the presence of several other potential isomers next to the evaluated highest hydroxybutyrfentanyl metabolites. Fragment ions of the highest peak indicated hydroxylation of the alkyl side chain. However, the main focus of the presented work was not the elucidation of butyrfentanyl metabolism, but rather its postmortem behavior. Further structure elucidation was therefore omitted.

In contrast to fentanyl - where norfentanyl is the most abundant metabolite in blood [4, 7] hydroxybutyrfentanyl and carboxybutyrfentanyl showed highest abundance in this butyrfentanyl case. A possible reason for the higher abundance of the carboxy-metabolite in the butyrfentanyl metabolism might be the longer alkyl chain in comparison to fentanyl or 3-methylfentanyl, which might enhance interaction with alcohol and aldehyde dehydrogenases.

As expected, the metabolite ratios in adipose tissue were relatively low, which can be explained by their higher hydrophilicity compared to the parent compound. The rather high metabolite abundance in liver, kidney and urine correlates with the higher abundance of metabolizing enzymes in these organs and their excretion pathway. Interestingly, the metabolite ratio of carboxybutyrfentanyl in brain was lower compared to all other matrices and compared to hydroxy- and desbutyrfentanyl. This low abundance in brain could be explained by the higher hydrophilicity of carboxybutyrfentanyl compared to the other metabolites, which might have prevented its crossing of the blood brain barrier (BBB). Additionally, it does not seem to be transported extensively. However, it is not clear whether hydroxy- and desbutyrfentanyl entered the brain by crossing the $\mathrm{BBB}$ or if direct formation in the brain could have occurred. Comparison of the relative metabolite concentrations at $\mathrm{t} 1$ and $\mathrm{t} 2$ also showed apparent concentration changes. Especially norbutyrfentanyl was prone to great concentration losses between the time points and was not detectable anymore in several matrices at $\mathrm{t} 2$. It therefore stays unclear, whether norbutyrfentanyl is a minor metabolite compared to hydroxy- or carboxybutyrfentanyl or if its low concentrations resulted from postmortem degradation or redistribution processes. However, the mechanisms behind the concentration changes could not be explained so far. 


\section{Conclusion}

A fatal case of butyrfentanyl poisoning revealed relevant postmortem concentration increases of butyrfentanyl in femoral and heart blood within 19 hours after first sampling. Highest abundant metabolites were hydroxybutyrfentanyl and carboxybutyrfentanyl. While hydroxybutyrfentanyl concentration stayed constant in peripheral blood, carboxybutyrfentanyl concentration decreased. Consequently, postmortem butyrfentanyl concentrations should be interpreted with great caution because of PMR processes. 


\section{Artwork and Tables}

Table 1 Chemical formulas, exact masses and structures of butyrfentanyl and its metabolites

\begin{tabular}{|c|c|c|c|}
\hline Analyte & Chemical formula & Exact mass $[\mathbf{M}+\mathbf{H}]^{+}$ & Structure \\
\hline Butyrfentanyl & $\mathrm{C}_{23} \mathrm{H}_{30} \mathrm{~N}_{2} \mathrm{O}$ & 351.2431 & \\
\hline Carboxybutyrfentanyl & $\mathrm{C}_{23} \mathrm{H}_{28} \mathrm{~N}_{2} \mathrm{O}_{3}$ & 381.2173 & \\
\hline Hydroxybutyrfentanyl & $\mathrm{C}_{23} \mathrm{H}_{30} \mathrm{~N}_{2} \mathrm{O}_{2}$ & 367.2380 & \\
\hline Desbutyrfentanyl & $\mathrm{C}_{19} \mathrm{H}_{24} \mathrm{~N}_{2}$ & 281.2012 & \\
\hline Norbutyrfentanyl & $\mathrm{C}_{15} \mathrm{H}_{22} \mathrm{~N}_{2} \mathrm{O}$ & 247.1805 & \\
\hline
\end{tabular}

Table 2 Butyrfentanyl LC-MS/MS method validation results

\begin{tabular}{ccccccc} 
QC & $\begin{array}{c}\text { Concentration } \\
{[\mathbf{n g} / \mathbf{m L}]}\end{array}$ & Calibration & $\begin{array}{c}\text { Precision } \\
{[\%]}\end{array}$ & $\begin{array}{c}\text { Accuracy } \\
{[\%]}\end{array}$ & $\begin{array}{c}\text { Matrix effect } \\
\mathbf{\pm} \text { RSD [\%] }\end{array}$ & $\begin{array}{c}\text { Matrix effect } \\
\mathbf{\pm} \text { RSD [\%] } \\
\text { IS corrected }\end{array}$ \\
\hline Low & 2.0 & ${ }^{12} \mathrm{C}$ & 21 & 97 & $75 \pm 15$ & $93 \pm 3.8$ \\
Med & 300 & ${ }^{12} \mathrm{C}$ & 4.1 & 108 & - & - \\
\hline Med & 300 & ${ }^{13} \mathrm{C}$ & 4.3 & 115 & - & - \\
High & 2250 & ${ }^{13} \mathrm{C}$ & 4.6 & 94 & $106 \pm 10$ & $104 \pm 2.9$ \\
\hline
\end{tabular}

Table 3 Butyrfentanyl concentration ratios between the matrices and femoral blood. Unavailable samples are indicated by “_-“.

\begin{tabular}{l|cc} 
Matrix & $\mathbf{t 1}$ & $\mathbf{t 2}$ \\
\hline Heart blood right ventricle & 0.6 & 0.4 \\
Heart blood left ventricle & - & 0.5 \\
Urine & - & 7.6 \\
Muscle & 1.6 & 1 \\
Liver l. dexter & 0.9 & 0.5
\end{tabular}




\begin{tabular}{l|cc} 
Liver l. sinister & - & 0.6 \\
Kidney & 2.4 & 1.3 \\
Lung & 46 & 14 \\
Spleen & 8.8 & 3.3 \\
Adipose tissue & 8.3 & 5.5 \\
Gastric content & - & 14 \\
Frontal lobe & - & 1.5 \\
Thalamus & - & 1.4 \\
Cerebellum & - & 1.6 \\
Centrum semiovale & - & 2.3 \\
Praecuneus & - & 1.6 \\
Hair & - & 75
\end{tabular}

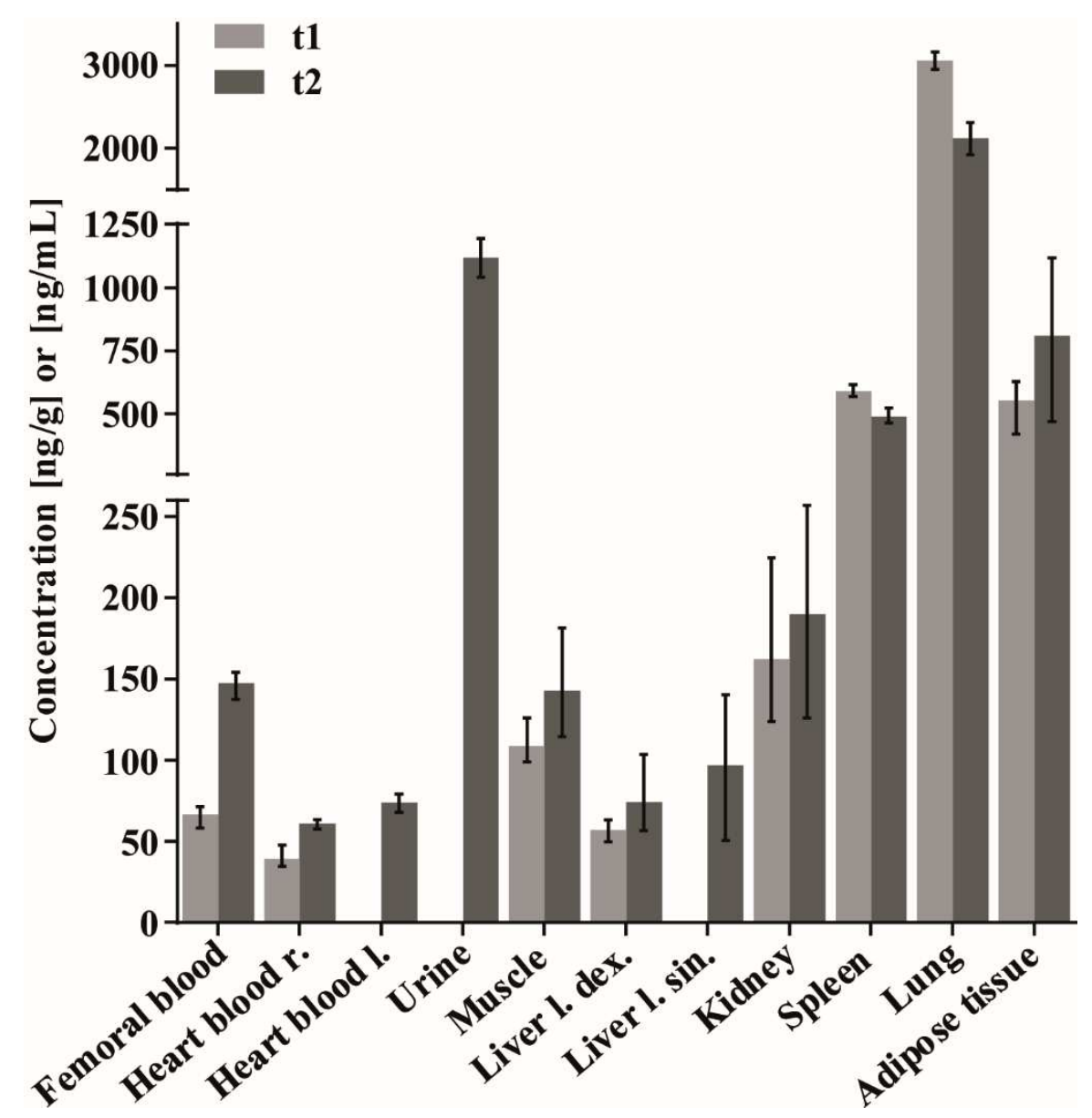

Figure 1 Butyrfentanyl mean concentration and range of triplicate measurements at $\mathrm{t} 1$ (light grey) and t2 (dark grey) 


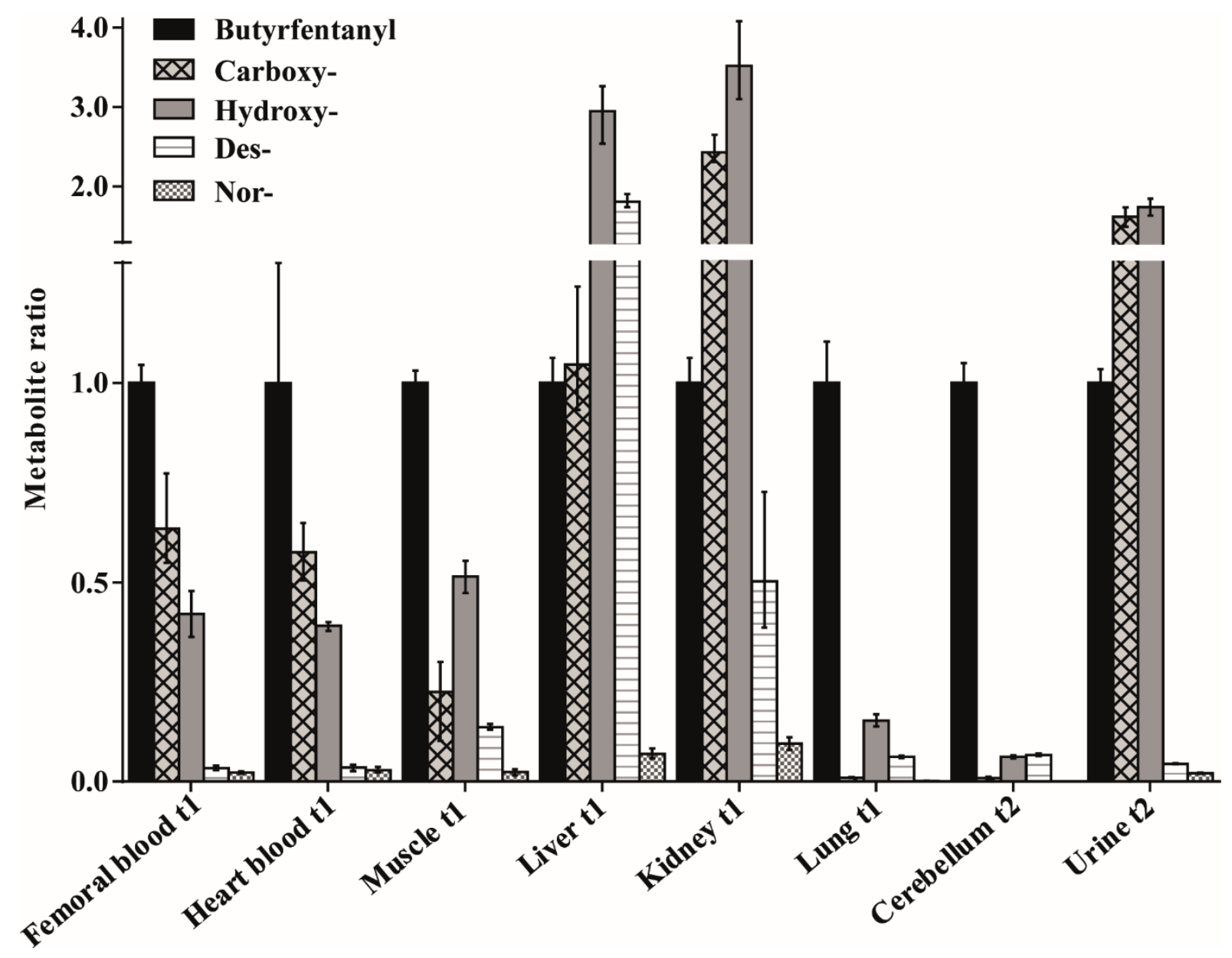

Figure 2 Mean metabolite ratios (relative metabolite concentration divided by mean butyrfentanyl relative concentration) and range ( $\mathrm{n}=3)$ in femoral blood, heart blood, muscle, liver, kidney and lung tissue at $\mathrm{t} 1$ and in cerebellum and urine at $\mathrm{t} 2$. 


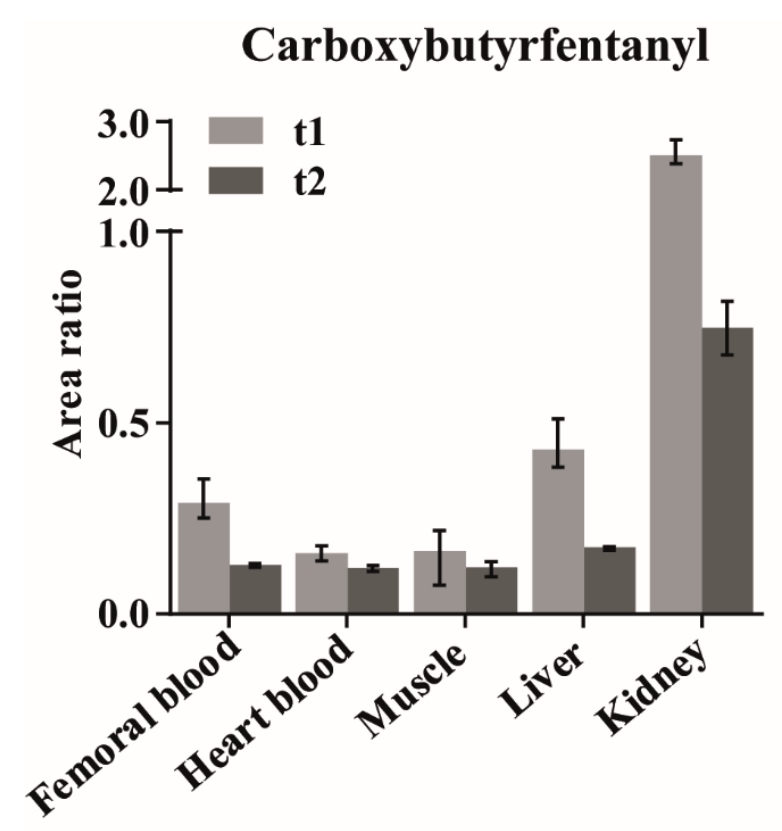

\section{Desbutyrfentanyl}

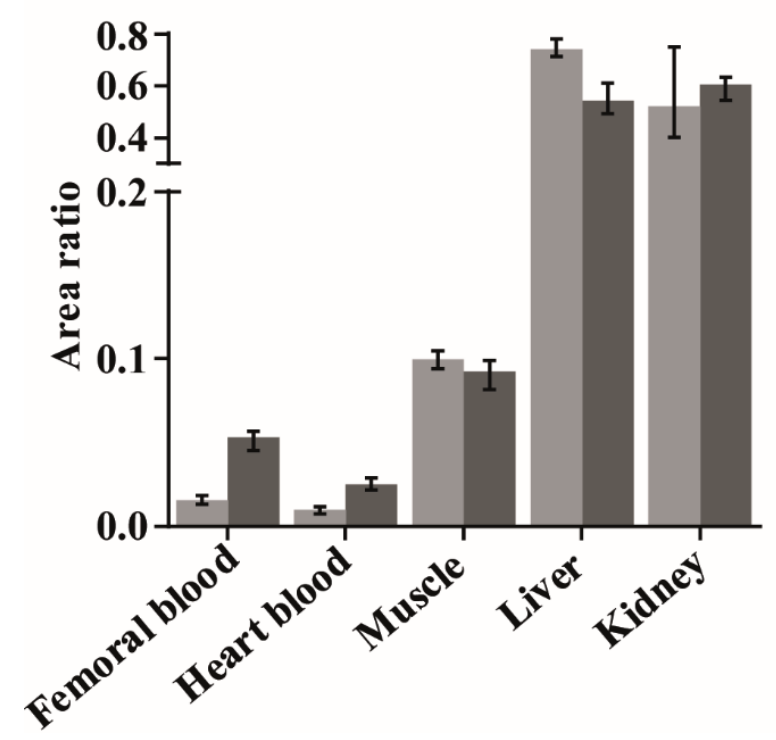

Hydroxybutyrfentanyl
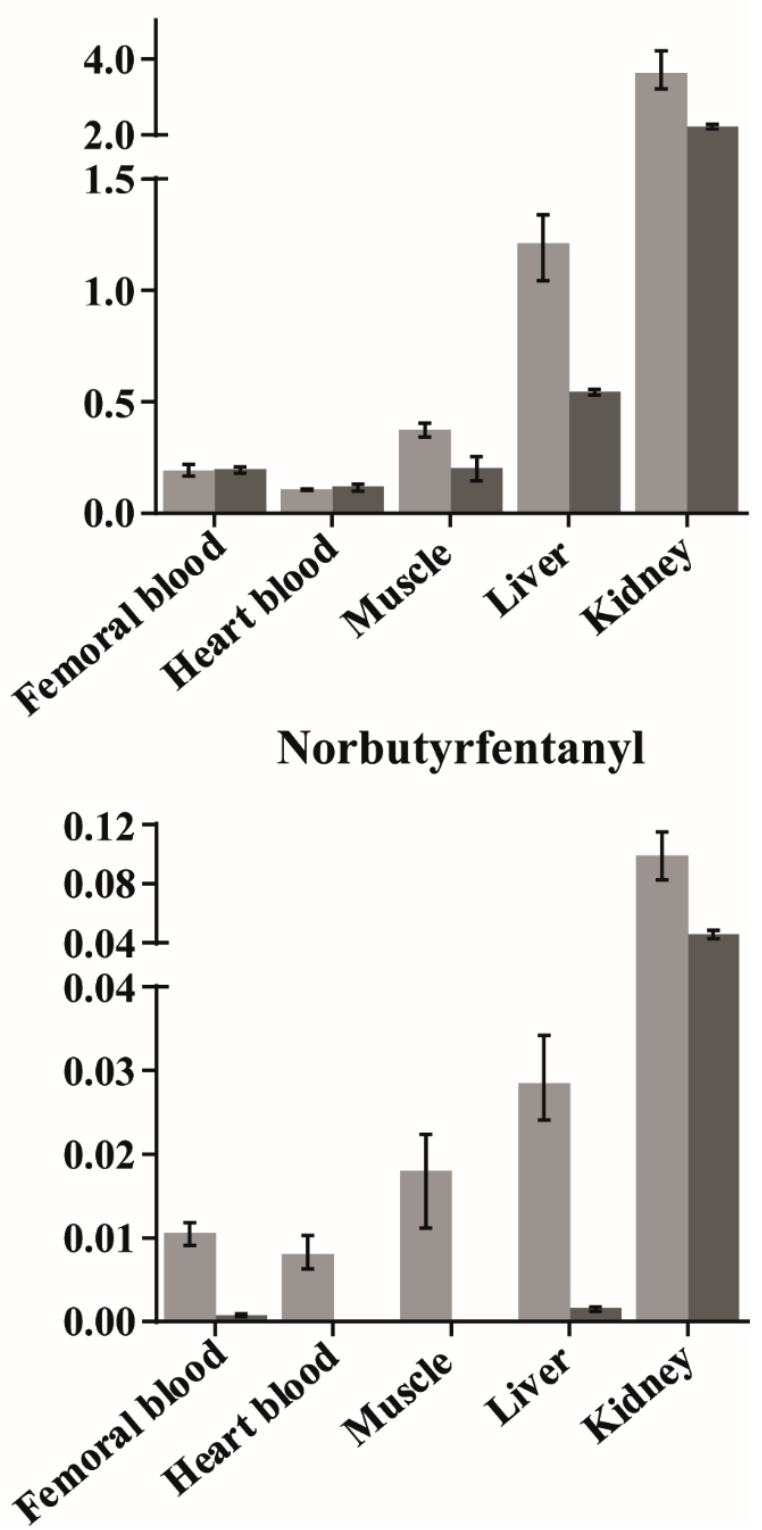

Figure 3 Relative concentration changes of the metabolites between $\mathrm{t} 1$ and $\mathrm{t} 2$ displayed as mean relative concentration and range at $\mathrm{t} 1$ and $\mathrm{t} 2$ exemplified for femoral and heart blood, muscle, liver and kidney 


\section{References}

[1] European drug report 2015 - trends and developments. Lisbon: European Monitoring Centre for Drugs and Drug Addiction (EMCDDA); 2015.

[2] Poklis J, Poklis A, Wolf C, Mainland M, Hair L, Devers K, et al. Postmortem tissue distribution of acetyl fentanyl, fentanyl and their respective nor-metabolites analyzed by ultrahigh performance liquid chromatography with tandem mass spectrometry. Forensic Sci Int. 2015;257:435-41.

[3] Ojanpera I, Gergov M, Liiv M, Riikoja A, Vuori E. An epidemic of fatal 3-methylfentanyl poisoning in Estonia. Int J Legal Med. 2008;122:395-400.

[4] Baselt RC. Disposition of toxic drugs and chemicals in man. 8th ed. Foster City CA: Chemical Toxicology Institute; 2008.

[5] Higashikawa Y, Suzuki S. Studies on 1-(2-phenethyl)-4-(N-propionylanilino)piperidine (fentanyl) and its related compounds. VI. Structure-analgesic activity relationship for fentanyl, methyl-substituted fentanyls and other analogues. Forensic Toxicol. 2008;26:1-5.

[6] Meyer MR, Dinger J, Schwaninger AE, Wissenbach DK, Zapp J, Fritschi G, et al. Qualitative studies on the metabolism and the toxicological detection of the fentanyl-derived designer drugs 3-methylfentanyl and isofentanyl in rats using liquid chromatography-linear ion trap-mass spectrometry (LC-MS(n)). Anal Bioanal Chem. 2012;402:1249-55.

[7] Labroo RB, Paine MF, Thummel KE, Kharasch ED. Fentanyl metabolism by human hepatic and intestinal cytochrome P450 3A4: implications for interindividual variability in disposition, efficacy, and drug interactions. Drug Metab Dispos. 1997;25:1072-80.

[8] McIntyre IM, Trochta A, Gary RD, Malamatos M, Lucas JR. An acute acetyl fentanyl fatality: a case report with postmortem concentrations. J Anal Toxicol. 2015;39:490-4.

[9] McIntyre IM, Trochta A, Gary RD, Wright J, Mena O. An acute butyr-fentanyl fatality: a case report with postmortem concentrations. J Anal Toxicol. 2016;40:162-6.

[10] Backberg M, Beck O, Jonsson KH, Helander A. Opioid intoxications involving butyrfentanyl, 4fluorobutyrfentanyl, and fentanyl from the Swedish STRIDA project. Clin Toxicol (Phila). 2015;53:609-17.

[11] Cole JB, Dunbar JF, McIntire SA, Regelmann WE, Slusher TM. Butyrfentanyl overdose resulting in diffuse alveolar hemorrhage. Pediatrics. 2015;135:e740-3.

[12] Skopp G. Postmortem toxicology. Forensic Sci Med Pathol. 2010;6:314-25.

[13] Prouty RW, Anderson WH. The forensic science implications of site and temporal influences on postmortem blood-drug concentrations. J Forensic Sci. 1990;35:243-70.

[14] Pounder DJ, Adams E, Fuke C, Langford AM. Site to site variability of postmortem drug concentrations in liver and lung. J Forensic Sci. 1996;41:927-32.

[15] Hilberg T, Rogde S, Morland J. Postmortem drug redistribution--human cases related to results in experimental animals. J Forensic Sci. 1999;44:3-9.

[16] Yarema MC, Becker CE. Key concepts in postmortem drug redistribution. Clin Toxicol (Phila). 2005;43:235-41.

[17] Han E, Kim E, Hong H, Jeong S, Kim J, In S, et al. Evaluation of postmortem redistribution phenomena for commonly encountered drugs. Forensic Sci Int. 2012;219:265-71.

[18] Gerostamoulos D, Beyer J, Staikos V, Tayler P, Woodford N, Drummer OH. The effect of the postmortem interval on the redistribution of drugs: a comparison of mortuary admission and autopsy blood specimens. Forensic Sci Med Pathol. 2012;8:373-9.

[19] Saar E, Beyer J, Gerostamoulos D, Drummer OH. The time-dependant post-mortem redistribution of antipsychotic drugs. Forensic Sci Int. 2012;222:223-7.

[20] Gerostamoulos J, Drummer OH. Postmortem redistribution of morphine and its metabolites. J Forensic Sci. 2000;45:843-5.

[21] Olson KN, Luckenbill K, Thompson J, Middleton O, Geiselhart R, Mills KM, et al. Postmortem redistribution of fentanyl in blood. Am J Clin Pathol. 2010;133:447-53.

[22] Ceelen L, De Zwart L, Voets M, Hillewaert V, Monbaliu J, Teuns G, et al. Postmortem redistribution of fentanyl in the rabbit blood. Am J Forensic Med Pathol. 2012;33:119-23.

[23] Staeheli SN, Gascho D, Fornaro J, Laberke P, Ebert LC, Martinez RM, et al. Development of CT-guided biopsy sampling for time-dependent postmortem redistribution investigations in blood and alternative matricesproof of concept and application on two cases. Anal Bioanal Chem. 2016;408:1249-58.

[24] Maurer HH, Pfleger K, Weber AA. Mass spectral and GC data of drugs, poisons, pesticides, pollutants and their metabolites. Weinheim: Wiley-VCH; 2011.

[25] Maurer HH, Wissenbach DK, Weber AA. Maurer/Wissenbach/Weber LC-MSn library of drugs, poisons and their metabolites: Wiley-VCH; 2014.

[26] Steuer AE, Forss AM, Dally AM, Kraemer T. Method development and validation for simultaneous quantification of 15 drugs of abuse and prescription drugs and 7 of their metabolites in whole blood relevant in 
the context of driving under the influence of drugs--usefulness of multi-analyte calibration. Forensic Sci Int. 2014;244:92-101.

[27] Staeheli SN, Poetzsch M, Kraemer T, Steuer AE. Development and validation of a dynamic range-extended LC-MS/MS multi-analyte method for 11 different postmortem matrices for redistribution studies applying solvent calibration and additional (13)C isotope monitoring. Anal Bioanal Chem. 2015;407:8681-712.

[28] Roemmelt AT, Steuer AE, Poetzsch M, Kraemer T. Liquid chromatography, in combination with a quadrupole time-of-flight instrument (LC QTOF), with sequential window acquisition of all theoretical fragment-ion spectra (SWATH) acquisition: systematic studies on its use for screenings in clinical and forensic toxicology and comparison with information-dependent acquisition (IDA). Anal Chem. 2014;86:11742-9.

[29] Madry MM, Steuer AE, Vonmoos M, Quednow BB, Baumgartner MR, Kraemer T. Retrospective monitoring of long-term recreational and dependent cocaine use in toenail clippings/scrapings as an alternative to hair. Bioanalysis. 2014;6:3183-96.

[30] Peters FT, Drummer OH, Musshoff F. Validation of new methods. Forensic Sci Int. 2007;165:216-24.

[31] Schutz H, Paine A, Erdmann F, Weiler G, Verhoff MA. Immunoassays for drug screening in urine : Chances, challenges, and pitfalls. Forensic Sci Med Pathol. 2006;2:75-83.

[32] Kaufmann AM, Krise JP. Lysosomal sequestration of amine-containing drugs: analysis and therapeutic implications. J Pharm Sci. 2007;96:729-46.

[33] Daniel WA. Mechanisms of cellular distribution of psychotropic drugs. Significance for drug action and interactions. Prog Neuropsychopharmacol Biol Psychiatry. 2003;27:65-73.

[34] Andresen H, Gullans A, Veselinovic M, Anders S, Schmoldt A, Iwersen-Bergmann S, et al. Fentanyl: toxic or therapeutic? Postmortem and antemortem blood concentrations after transdermal fentanyl application. J Anal Toxicol. 2012;36:182-94.

[35] McIntyre IM. Liver and peripheral blood concentration ratio (L/P) as a marker of postmortem drug redistribution: a literature review. Forensic Sci Med Pathol. 2014;10:91-6.

[36] Moriya F, Hashimoto Y. Redistribution of basic drugs into cardiac blood from surrounding tissues during early-stages postmortem. J Forensic Sci. 1999;44:10-6. 